\title{
Has the lung cancer risk from smoking increased over the last fifty years?
}

\author{
David M. Burns • Christy M. Anderson • \\ Nigel Gray
}

Received: 11 July 2010/Accepted: 30 November 2010/Published online: 25 December 2010

(C) The Author(s) 2010. This article is published with open access at Springerlink.com

\begin{abstract}
Background We examine whether the lung cancer risk due to smoking has increased over time.

Methods Lung cancer risk equations based on prospective mortality data collected from 1960 to 1972 were applied to 5 -year birth-cohort-specific estimates of smoking behaviors among white males to estimate lung cancer mortality rates for U.S. white males from 1960 to 2000. These estimated rates were compared to U.S. white male mortality rates for the same birth cohorts.

Results Observed birth-cohort-specific U.S. lung cancer mortality rates are substantially higher than those expected from changes in smoking behaviors, and the proportional difference increases with advancing calendar year. This trend persisted even when the duration term was increased in the risk equation. However, adjusting for changes in cigarette design over time by adding a term for the duration of smoking after 1972 resulted in the predicted rates closely approximating the observed U.S. mortality rates.

Conclusion Lung cancer risk estimates observed during the 1960s under predict current lung cancer mortality rates in U.S. white males. Adjustment for the duration of smoking after 1972 results in estimates that reasonably approximate the observed U.S. lung cancer mortality, suggesting that lung cancer risks from smoking are increasing in the United States coincident with changes in cigarette design.
\end{abstract}

D. M. Burns $(\square)$. C. M. Anderson

UCSD School of Medicine, 1120 Solana Dr, Del Mar, San

Diego, CA, USA

e-mail: dburns@ucsd.edu

N. Gray

Cancer Council of Victoria, Melbourne, VIC, Australia
Keywords Cigarettes - Lung cancer - Changing risk · Changing cigarettes

\section{Introduction}

Cigarette smoking is responsible for $85-90 \%$ of the lung cancer occurring in the United States [1]. Epidemiological data suggest that the risks of smoking for lung cancer may be increasing. Risks for lung cancer among smokers in the 6-year follow-up of the Cancer Prevention Study II (CPS II) begun in 1982 and are substantially higher than those observed among smokers two decades earlier in the Cancer Prevention Study I (CPS I) which began in 1959, even when differences in intensity and duration of smoking are controlled in the analyses [2]. Risks for never smokers were similar in the two studies [3] and have remained unchanged over time [4]. An increased risk of smoking for lung cancer is also observed when the second 20 years of follow-up of the British Physicians study is compared to the first 20 years [5]. Changes in cigarette design and smoke composition over the past several decades have been suggested as a likely cause of this increase in the lung cancer risk of smoking $[2,6,7]$.

We hypothesize that lung cancer risks due to cigarette smoking have increased over the period from 1960 to 2000 coincident with known changes in cigarette design [8]. Data from the 12-year follow-up of CPS I provide smoking behavior-based predictions of lung cancer mortality risks $[9,10]$ using observations of lung cancer risk obtained during the 1960s. These risk equations are applied to 5-year birth-cohort-specific estimates of smoking behaviors for U.S. white males over time in order to estimate the changes in lung cancer rates expected due to changes in smoking behaviors. We compare the resulting estimates to observed 
U.S. national lung cancer mortality rates for the same cohorts in the calendar years from 1960 to 2000. Differences between the estimated and observed lung cancer rates are examined for trends across calendar year.

White males were selected for these analyses because CPS I has substantially fewer lung cancer deaths among women, or among African Americans, making the development of risk equations for smoking behaviors in the 1960s for these groups less reliable and more difficult to interpret.

\section{Methods}

Lung cancer risk model

We use published risk equations derived from the lung cancer mortality experience of white males in the 12-year follow-up of CPS I to estimate lung cancer risks for active smokers. These equations have terms for age, duration of smoking, and number of cigarettes smoked per day $[9,10]$. Excess risk equations based on CPS I data extend these risk equations to include risks for former and never smokers [9]. The risks for never smokers, current smokers, and former smokers can be summed to generate an estimate of the lung cancer death rate for a 5-year birth cohort of white males by single calendar year once smoking behaviors and age for the birth cohort are specified.

\section{Birth-cohort-specific estimates of smoking behavior}

Estimates of smoking initiation, prevalence, and cessation among white males by 5 -year birth cohorts born between 1900 and 1954 were developed for individual calendar years in conjunction with the National Cancer Institute Cancer Intervention and Surveillance Modeling Network (CISNET). A database combining questions on smoking behavior from all of the National Health Interview Surveys from 1965 to 2005 and methods previously described [11, 12] is used to generate the estimates. For each calendar year, these estimates provide rates of smoking initiation, prevalence of current and former smoking, distributions of duration of smoking, duration of abstinence and number of cigarettes smoked per day for current and former smokers, and the duration of abstinence for former smokers. These smoking behaviors are used to estimate lung cancer death rates using the lung cancer risk equations.

Actual and estimated lung cancer mortality rates

Observed mortality rates for cancer of the lung and bronchus among white males are obtained from the National
Center for Health Statistics (NCHS) by single year of age and by single calendar year from 1969 to 2003 . Rates for a 5 -year birth cohort were generated for 5-year birth cohorts beginning with 1900-1904 and ending with 1950-1954 birth cohort. For the years from 1960 to 1968 , we used the lung cancer mortality rates for white males provided by Mannino, et al. [13]. Since Mannino used 5-year birth cohorts that differed from ours by 1 year, the rates were adjusted by performing regression modeling of the lung cancer mortality rates from the NCHS against the Mannino rates for the overlapping calendar years of 1969-1994.

Estimated lung cancer mortality rates are generated by applying the risk equations for never, current, and former smokers to the population estimates of smoking behavior for each calendar year. The smoking behaviors are arranged in cells specific for smoking status, duration of smoking, duration of abstinence, and cigarettes smoked per day. Lung cancer mortality rates are estimated for each cell using the appropriate risk equation. A summary rate for the entire cohort in a given year is generated as the average (weighted by the fraction of the population in the cell) of the rates in all of the cells for that year.

Adjustments to the estimates

\section{Difference between the mortality in CPS I and U.S. mortality}

Lung cancer mortality for a healthy population such as that in CPS I is expected to be lower than that for the general U.S. Population, sometimes referred to as a healthy population selection bias[14]. We use a power function to adjust the predicted mortality rates upward to match the estimated mortality rates to the U.S. mortality. We estimate the parameters for this function by fitting the predicted rates $(\hat{R})$ to the observed rates $(R)$ using nonlinear regression to derive $\alpha_{1}$ and $\beta_{1}$ :

$R=\alpha_{1} \hat{R}^{\beta_{1}}$

\section{Differences due to increases in the risk of smoking}

We also perform an adjustment to capture the potential effect of an increased risk in smoking after time $t_{0}$. We scale upward the risk of lung cancer for continuing smokers and former smokers by incorporating a term for the number of years smoked after $t_{0}$ :

$\hat{R}_{S}^{\prime}=\left[\gamma\left(t-t_{0}\right)\right]^{I_{t}} \hat{R}_{S}$

where

$I_{t}= \begin{cases}1, & \gamma\left(t-t_{0}\right) \geq 1 \\ 0, & \gamma\left(t-t_{0}\right)<1\end{cases}$ 
$\hat{R}_{S}=$ the lung cancer risk estimated from the CPS 1 risk equations for continuing smokers; $t=$ current calendar year.

Since the adjustment for duration of smoking after $t_{0}$ is only used in the equation when it is greater than or equal to 1 , the adjustment can only increase the predicted rate of lung cancer mortality.

The analogous equations for former smokers are

$\hat{R}_{F S}^{\prime}=\left[\gamma\left(t-t_{0}-t_{Q}\right)\right]^{I_{t}} \hat{R}_{F S}$

where

$I_{t}= \begin{cases}1, & \gamma\left(t-t_{0}-t_{Q}\right) \geq 1 \\ 0, & \gamma\left(t-t_{0}-t_{Q}\right)<1\end{cases}$

$\hat{R}_{\mathrm{FS}}=$ the lung cancer risk estimated from the CPS 1 risk equations for former smokers; $t_{Q}=$ the year in which the former smoker quit smoking.

The predicted lung cancer mortality rate for the cohort in a specified year is adjusted for the difference between the mortality in CPS I and that for the U.S. population using the power function described earlier with the resulting equation being:

$R=\alpha_{2}\left(\hat{p}_{\mathrm{NS}} \hat{R}_{\mathrm{NS}}+\hat{p}_{S} \hat{R}_{\mathrm{S}}^{\prime}+\hat{p}_{\mathrm{FS}} \hat{R}_{\mathrm{FS}}^{\prime}\right)^{\beta_{2}}$

Nonlinear regression is used to estimate the parameters $\alpha_{2}$, $\beta_{2}$, and $\gamma$ simultaneously. Estimation of the parameters was performed manually by iterating $t_{0}$ in 5 -year increments while estimating one set of $\alpha_{2}, \beta_{2}$, and $\gamma$ for each increment until the variance, estimated by the mean squared error, was minimized.

\section{Results}

The question of whether the risks of smoking have increased over time is examined by generating birthcohort-specific lung cancer death rates for white males based on the risks observed during the 1960s (CPS I) and the changes in smoking behaviors for white males over the interval 1960-2000. As would be expected [14], the lung cancer death rates predicted from the smoking specific risks observed in the somewhat healthier population followed in the CPS I are lower than those observed in the U.S. mortality data. However, the pattern of underestimation varies with calendar year and birth cohort. Figure 1 presents ratios of the observed U.S. lung cancer death rates to those estimated for the same birth cohort in the same calendar year. These ratios are presented by single calendar year and mean year of age for the cohorts in Fig. 1.

The data in Fig. 1 demonstrate increases in the ratios with advancing calendar year and age. In both the age and calendar year plots, the data segregate by birth cohort with the most recent cohorts having the lowest ratios in the calendar year plot and the highest ratios in the age plot. Neither age nor calendar year seems to fully explain the difference by birth cohort.

The data in Fig. 1 are for all 5-year birth cohorts born after 1900. In order to minimize the potential contribution of over-reporting of duration of exposure in the earliest cohorts and to minimize the effects of the adjustment of differential mortality on ever smoking prevalence, we limit subsequent analyses to birth cohorts born after 1915 .
Fig. 1 Ratio of observed to predicted age-specific, and calendar year-specific lung cancer death rates for 5-year birth cohorts born 1900-1954
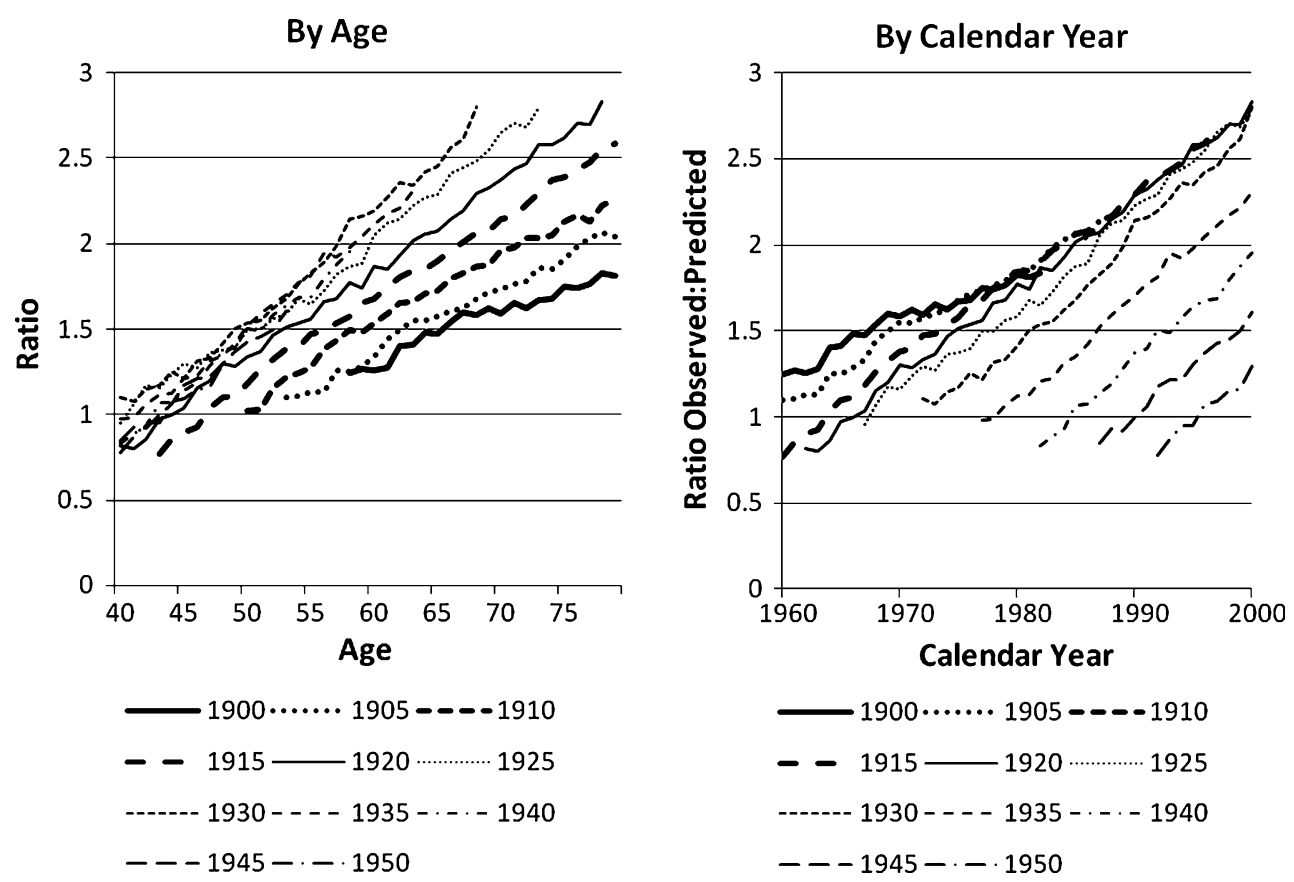
Adjustments for mortality differences between CPS I and the U.S. mortality

We initially explore whether adjusting for the expected healthy population bias in the CPS I data reduce the trends with age and calendar year seen in Fig. 1. This difference might be expected to be disproportionately larger at older ages where high rates of disease occur, and so we use a power function to make this adjustment with the equation:

$R=\alpha_{1} \hat{R}^{\beta_{1}}$

The birth cohort data for estimated lung cancer death rates $(\hat{R})$ are fit to the observed rates $(R)$ to derive the parameters $\alpha_{1}$ and $\beta_{1}$ using all of the calendar years available for the birth cohorts born after 1915 .

Adjustment for a healthy population bias using this approach reduces the absolute difference in lung cancer mortality rates, but substantive trends remained by calendar year and birth cohort. Figure 2 presents standardized residuals of the differences between these adjusted

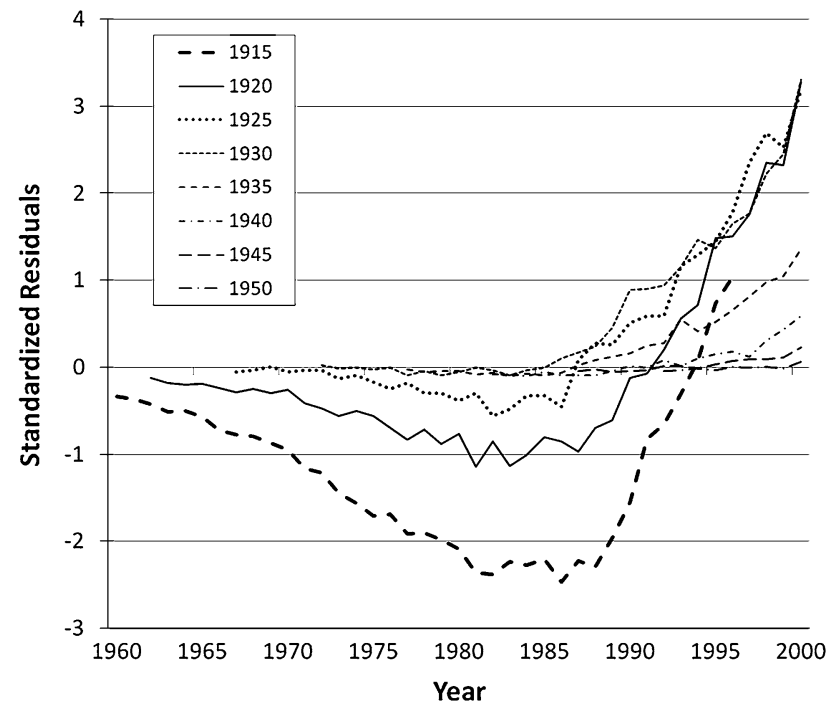

Fig. 2 Standardized residuals for the differences between estimated and observed white male lung cancer mortality rates by birth cohort after adjusting for a healthy population bias estimates and the observed lung cancer U.S. mortality rates by calendar year, and it is evident that substantive differences remain across birth cohorts and that there is a marked trend from negative to positive residuals with advancing calendar year after approximately 1980.

Adjusting for differences with calendar year

We next examined whether adjusting for an increasing toxicity over time of the cigarettes smoked could explain or reduce the trends with time observed in Figs. 1 and 2. Examination of the data in Fig. 1 by calendar year suggests that there is a shift of approximately 5 years in the point where the lines cross a value of 1 for sequential birth cohorts born after 1930, and when the values for the ratios are plotted by the duration of smoking likely to have occurred after 1945 or 1950, the lines for the different birth cohorts are roughly superimposed on one another (data not shown). This observation led us to examine whether adjusting for the duration of smoking after a defined calendar year, as might be expected from the changes in cigarette design, could explain the time trends seen in Figs. 1 and 2.

In order to keep the adjustment for calendar year as simple as possible, we introduced a single additional term into the risk equations used to estimate the increase in risks for both current and former smokers. That term is a constant $\gamma$ which is multiplied by the number of years of smoking after a specified calendar year and is therefore a proxy for the cumulative contribution of cigarettes smoked after that date to lung cancer risk. For example, someone born in 1915 who started to smoke in 1930 would have 50 years of smoking duration in 1980, but their duration of smoking after a specified year of 1945 would be only 35 years. The specified trigger year and the value of $\gamma$ are required to be identical for current smokers and former smokers in the risk estimation. In addition, since the intent of this adjustment was to increase the estimated risk, the adjustment was only applied when the term $\gamma^{*}$ durationafter-trigger-year exceeded a value of one.

Table 1 Parameter estimates for calendar year adjustment for different $t_{0}$

\begin{tabular}{|c|c|c|c|c|c|}
\hline \multirow[b]{2}{*}{ Parameter } & \multirow{2}{*}{$\begin{array}{l}t_{0}=1,940 \\
\text { Estimate }\end{array}$} & \multicolumn{2}{|c|}{$t_{0}=1,945^{\mathrm{a}}$} & \multirow{2}{*}{$\begin{array}{l}t_{0}=1,950 \\
\text { Estimate }\end{array}$} & \multirow{2}{*}{$\begin{array}{l}t_{0}=1,955 \\
\text { Estimate }\end{array}$} \\
\hline & & Estimate & $95 \% \mathrm{CI}$ & & \\
\hline$\alpha$ & 0.95 & 0.78 & $(0.73-0.82)$ & 0.65 & 0.57 \\
\hline$\beta$ & 1.06 & 1.13 & $(1.11-1.14)$ & 1.17 & 1.20 \\
\hline$\gamma$ & 0.038 & 0.036 & $(0.035-0.037)$ & 0.038 & 0.042 \\
\hline MSE & 135.7 & 78.25 & & 92.59 & 153.3 \\
\hline
\end{tabular}

${ }^{a}$ Best fit 
The parameters which adjust for the mortality differences between CPS I and the U.S. mortality $\left(\alpha_{2}, \beta_{2}\right)$ and $\gamma$ which reflects the increasing toxicity of smoking were derived for each of four trigger years by fitting the estimated rates to the observed U.S. mortality rates. Values of the parameter estimates for four trigger years $\left(t_{0}\right)$ are presented in Table 1 . The trigger year with the best fit was 1945. Since the estimates are applied only when the term $-\gamma$ times the duration of smoking after the trigger year-exceeds a value of 1 , the adjustments are applied only after 1972 and 27 years duration of smoking.

The results of adjusting the estimated rates for an increase in the toxicity of cigarettes over time are presented in Fig. 3 for each 5-year birth cohort born between 1915 and 1949. Rates are presented by single calendar year for the unadjusted estimates based on the CPS I risk equations, for the estimated rates adjusted for both the healthy population bias and a calendar year effect, and for the observed U.S. mortality. With the inclusion of the adjustment for increasing toxicity over time, it appears that the estimates closely match the observed U.S. mortality.

Figure 4 is an identity plot comparing the adjusted estimated rates and the observed U.S. mortality rates for all of the cohorts born after 1915 for ages 40-79 and calendar

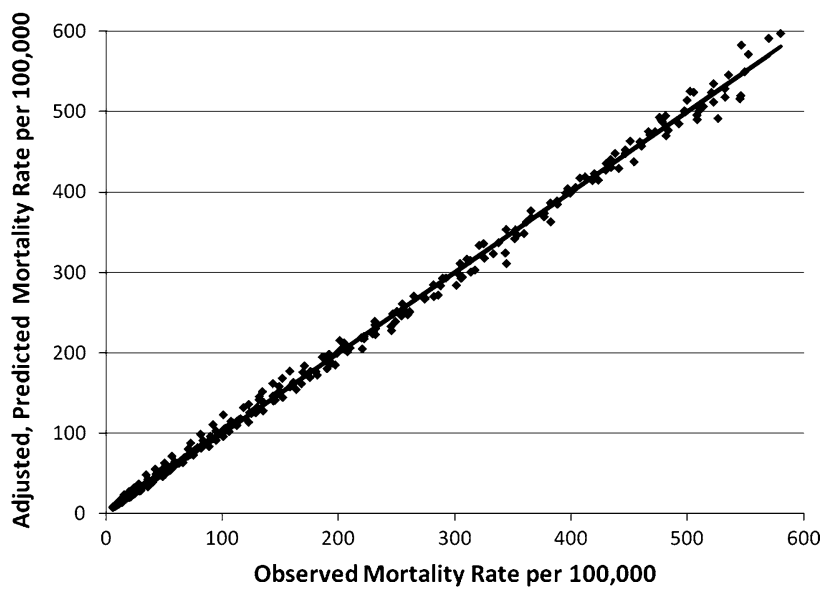

Fig. 4 Identity plot comparing predicted and observed lung cancer mortality rates

years 1960-2000. The values are closely grouped around the line of identity.

As might be expected from the data in Fig. 4, standardized residuals plotted against calendar year showed some oscillation around the zero value, but the birthcohort-specific pattern and trend of increasing residual with increasing calendar year evident in the unadjusted data are no longer evident.
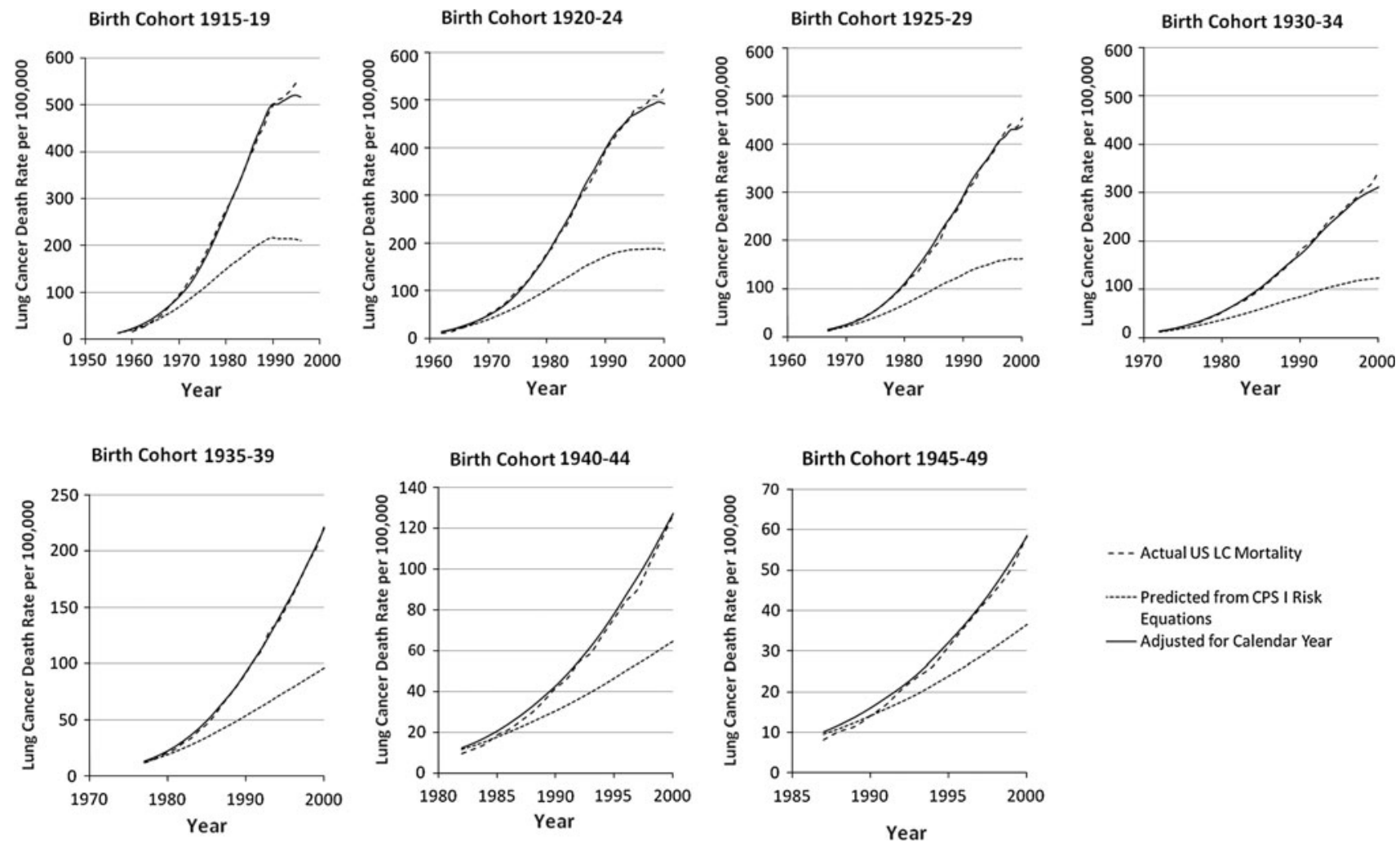

Fig. 3 Lung cancer death rates estimated from CPS I risk equations before and after adjusting for calendar year effects compared to the observed U.S. mortality rates for 5-year birth cohorts of white males by calendar year 
These results suggest that a simple arithmetic adjustment for the duration of smoking after 1972 (i.e., a trigger year of 1945 plus 27 years) results in a reasonable match between the estimates derived and the observed U.S. mortality for lung cancer in white males.

Adjustments for underestimation of duration effects in CPS I

A potential alternate explanation for the increase in risk observed between CPS I and CPS II [2] is an underestimate of the effect of duration of smoking due to over-reporting of smoking duration in CPS I. The transition from other forms of tobacco use to cigarette use largely began after 1914 [11], and much of the lung cancer occurrence in CPS I was observed among individuals who reported initiating cigarette smoking before 1914. It is possible that some of these individuals may have reported their cigarette smoking duration as beginning with their first use of any form of tobacco leading to a longer reported duration of cigarette smoking than actually occurred. This erroneous reporting of a longer duration of smoking could lead to a reduced magnitude for the effect of duration of smoking on lung cancer risk in the CPS I risk equations. Since a much larger fraction of those who developed lung cancer in CPS II took up smoking after 1914, the effect of this form of overreporting of duration of smoking would be less, the magnitude of the duration term would increase, and the risk of smoking would appear to have increased between the two studies when adjusted for differences in reported duration of smoking. An underestimation of the magnitude of the contribution of duration of smoking to lung cancer risk could also lead to a progressively increasing underestimation of lung cancer rates for the U.S. population as calendar year advanced and the cumulative duration of smoking in the population increased.

We examined the potential for an underestimation of the effect of duration on risk to explain our observation of progressively increasing underestimate of U.S. mortality with advancing calendar year. We systematically altered the exponent for the duration term in 0.1 increments and decrements and compared the new estimates for birthcohort-specific risks to the observed U.S. mortality rates using the previously derived values for the adjustments for the differences between CPS I and the U.S. mortality (the $\alpha_{1}$ and $\beta_{1}$ ) parameters. The best fit for the duration increments (2.9) varied little from the one derived directly from the CPS I data (2.87), and the pattern of the residuals continue to demonstrate clear cohort effects and progressive increases in the standardized residuals with increasing calendar year.

We also examined this question by selecting the best fitting increment in the duration exponent without utilizing the $\alpha_{1}$ and $\beta_{1}$ parameters and then deriving new. $\alpha_{1}$ and $\beta_{1}$

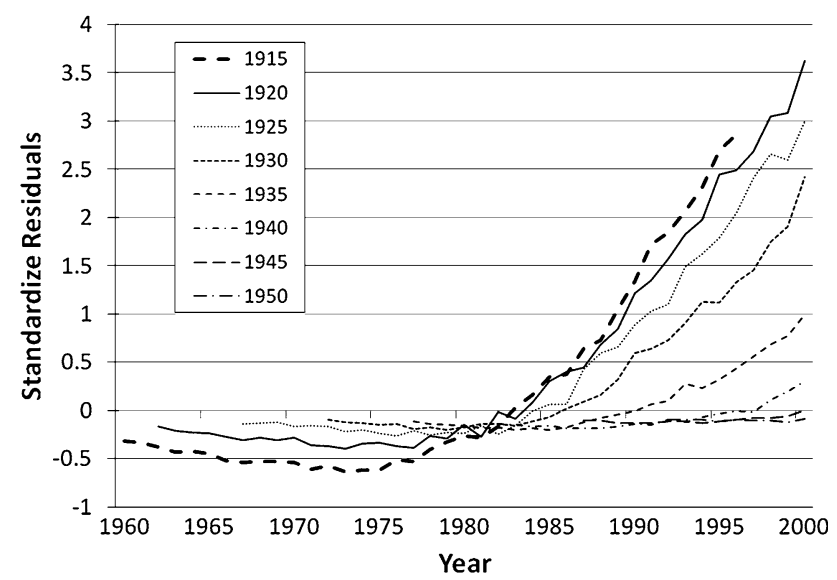

Fig. 5 Standardized residuals for the estimates from the CPS I risk equations with the duration exponent increased to 3.1 and new $\alpha_{1}$ and $\beta_{1}$ parameters

parameters by re-fitting the estimates to the observed lung cancer mortality rates. This resulted in a somewhat greater increase in the duration exponent producing the best fit (to 3.1 ), but the resulting residuals continued to display cohort and calendar year effects as presented in Fig. 5.

These results demonstrate that in contrast with adjustments for increasing toxicity of the cigarette smoked, simple adjustments of the duration effect in the CPS I risk equations do not provide reasonable estimates of the U.S. lung cancer mortality experience.

\section{Discussion}

It may be tempting for the reader to consider this paper as a presentation of a model for predicting U.S. lung cancer mortality. However, that is not our intent. Our intent is to take a set of simple risk equations shown to predict lung cancer rates well in a large epidemiological study conducted during the 1960s and examine the changes in how well the estimates from these risk equations continue to predict observed U.S. lung cancer mortality in white males over the subsequent decades. We then explore the effect of simple adjustments for those factors postulated to explain the difference in risk estimates between CPS I and CPS II and the under prediction of U.S. mortality rates including: a healthy population effect, changes in the risks of smoking over time, and an underestimate of the duration effect in the CPS I data. Of these, adjusting for an increase in the risk of smoking over time was the only simple adjustment which resulted in estimates that matched the changes in lung cancer risks for the U.S. population of white males over the past several decades.

Rates of lung cancer mortality among nonsmokers have been examined for changes over time and found to be 
relatively constant across calendar year [3, 4], suggesting that the changes in risk observed in our analyses are confined to smokers and are unlikely to be the result of changes in other risk factors for lung cancer.

Our goal is to further test the hypothesis that changes in cigarette design may have made smoking more dangerous over the past half century. We support that hypothesis by showing that even a simple adjustment of the CPS I risk equations for an increase in risk due to changes in cigarettes adequately predicts the observed mortality data. Adjustments for healthy population effects and for potential underestimates of the contribution of duration to risk are not able to eliminate either the differences in estimation accuracy across birth cohorts or the progressive underestimate of observed lung cancer mortality as calendar year advances.

The possibility that increasing lung cancer rates over time in birth cohort analysis is due to diagnostic advances has been examined by others and not felt to explain these trends $[7,15,16]$. While shifts in the age distribution of the population over time may contribute to increases in age adjusted lung cancer rates, they would not have a meaningful impact on the birth-cohort-specific rates we present. Since the estimates are based on the distribution of smoking behaviors at the start of each calendar year, differences in competing cause mortality over time will also have little effect. Shifts in the socioeconomic distribution of smokers over time could contribute to an increase in overall lung cancer death rates, but that shift should be relatively uniform across the birth cohorts and that is not what we observed. In addition, a companion analysis [17] of type-specific lung cancer risks demonstrates that the increase in lung cancer risk is predominantly for adenocarcinoma which is more difficult to explain as a socioeconomic effect. Recent data [18] also suggest that there may be a slower decline in death rates from adenocarcinoma of the lung than compared to squamous and small cell carcinomas, ant this may increase that proportion of all lung cancer that is adenocarcinoma as former smokers represent a larger proportion of the ever smoking population. Since our adjustment of the risk following cessation is based on the observed risks with different durations of abstinence in the CPS I follow-up, the effect of slower decline in adenocarcinoma rates would have been incorporated into the estimates of the overall decline in lung cancer rates. The limited analyses that address the issue of a shifting socioeconomic effect over time suggest that shifts in the social class of smokers has less effect on trends in adenocarcinoma than for the other types of lung cancer [19].

Models of risk based on smoking pattern have been applied by others to birth-cohort-specific smoking prevalence data in order to estimate the expected occurrence of lung cancer in the absence of any change in the risk of smoking. Swartz [20] used birth-cohort-specific smoking prevalence data and a multi-stage carcinogenesis model similar to that developed by Whittemore [21] to predict overall age-adjusted trends in lung cancer mortality for white males from 1970 to 1985 . He estimated that there should have been a $12 \%$ decline in rates over the interval based on the assumption of a constant effect over time in the risk model. This estimated decline contrasts sharply with the $26 \%$ increase in the observed lung cancer mortality rates over that interval. Tolley and colleagues [22] used an updated set of birth cohort smoking prevalence estimates, and a risk model developed by Peto [23], to predict lung cancer death rates over time by birth cohort. They estimated that overall lung cancer mortality should have begun to decline in the early 1980s for white males and in the mid-1990s for white females. Observed lung cancer mortality continued to rise throughout the $1980 \mathrm{~s}$ peaking in the early 1990s for white males [24] and may have only recently peaked for white females [25]. A similar approach using risk models developed from the CPS I data and birth-cohort-specific smoking prevalence data from the National Health Interview Survey (NHIS) demonstrated a systematic trend of increasing underestimation of observed lung cancer mortality rates across all birth cohorts with advancing calendar year [6]. None of these analyses included adjustment for changes in number of cigarettes smoked per day over time. We believe our analyses are the first to include the effect of differences in cigarettes smoked per day.

When considering the increase in lung cancer risk between CPS I and CPS, the possibility of underestimating both CPD and duration effects was considered [2]. Examination of patterns of per capita consumption and smoking behaviors in the early years of the last century suggest that cigarette smoking was uncommon in the U.S. prior to 1914, particularly in relation to other forms of tobacco use [11]. Since lung cancer is a disease of older ages, much of the lung cancer mortality experience in CPS I occurred among males who were well past their adolescence by 1914, and yet many of them report initiating smoking at early ages. It is therefore possible that some of these individuals reported initiating cigarette smoking at the time that they first used tobacco of any type or otherwise overestimated their duration of cigarette smoking. This may also result in an overestimation of the number of cigarettes smoked early in life.

Overestimation of duration of smoking in CPS I data would result in an underestimate of the contribution of duration to lung cancer risk in the risk equations. Since it is exponential in effect, it is not hard to contemplate that a substantive underestimate of the contribution duration from CPS I could lead to both an increase between CPS I and II 
and the increasing underestimate of U.S. mortality seen in our analyses. Nevertheless, our results suggest that is not that case. While increasing the duration term does increase the estimated rates, as it must, the pattern remains one where there is a progressive change as calendar year advances with an overestimate of the actual risk giving way to an underestimate of the risk. Simply increasing the contribution of the duration term does not resolve the changes over time. It is possible that more sophisticated approaches to modeling or more complex adjustments could resolve the temporal trend differences, but even the complex two stage clonal expansion models used by the NCI CISNET effort have required age period cohort adjustments to match U.S. mortality rates.

The contribution of cigarettes smoked per day to lung cancer risk is much smaller than the contribution of duration $[10,26]$, and the exponent for the cigarettes per day term in the CPS I risk equations is close to 1. As a result, underestimation of lifetime number of cigarettes smoked per day due to over-reporting of smoking early in life would be expected to make only a modest contribution to mis-estimation of the risk and that mis-estimation should largely be absorbed by the constant parameter used to adjust for healthy populations $\left(\alpha_{1}\right)$, since the effect would be approximated by a constant. The effect of an overestimate of duration in the CPS I data would be expected to be larger since the exponent on the duration term in the risk equation is large (2.87).

There have been substantial changes in cigarette design and manufacture over to past several decades [8] including the addition of filters to cigarettes, using ventilation holes in those filters to lower machine estimates of tar exposure, a substantial increase in the carcinogenic tobacco-specific nitrosamines in cigarettes [27] as well as other changes [8]. Ventilated filters increase the puff volume used by the smoker and may lead to deeper inhalation of the smoke increasing the smoke exposure to the lung [28]. Increasing nitrosamine levels may make the smoke more carcinogenic at any level of exposure. Widespread shift to filtered cigarettes occurred in the 1950s and changes in the nitrosamine levels date from the 1960s [8, 29]. Our data do not allow differentiation between these two potential mechanisms for an increased risk of smoking over time.

These analyses rely on risk equations, survey estimates of smoking behavior, and U.S. mortality data; and no individual level information linking smoking behaviors to lung cancer outcomes is utilized. As such, the results of our analyses can be used for hypothesis generation and support, but they should not be considered as proving causation. As with any analyses of this type, there is always a potential for misspecification or inaccuracy in the underlying data to confound the results.
Our data demonstrate an increase in the risk associated with smoking over the past several decades as estimated using risk equations developed from smokers during the 1960s and changes in smoking behaviors from national survey data. Adjustment of the risk equations for the duration of smoking after 1972 allows the estimates to reasonably approximate the observed U.S. lung cancer mortality experience, but simple adjustments for the effect of total duration of smoking does not. These results support the hypothesis that lung cancer risks from smoking may be increasing in the U.S. due to changes in cigarette design, and correspondingly that regulatory control over cigarette design may have the potential to reduce the risk of smoking. While not conclusive, our results suggest that the potential for increasing lung cancer risk from changes in cigarette design over the past half century should be explored more fully.

Acknowledgments We would like to acknowledge the advice and support of Michael Thun of the American Cancer Society in providing access to the CPS I data and reviewing the manuscript. This work was supported by contracts from the National Cancer Institute 263-MQ-320522 and 263-MQ-414669 and the Tobacco Control Research and Education Foundation.

Conflict of interest David Burns has testified extensively in tobacco litigation against the tobacco industry including in litigation on the risks of low tar cigarettes. The Tobacco Control Research and Education Foundation has received an unrestricted gift from attorneys who were involved in tobacco litigation against the tobacco industry that led to the Master Settlement Agreement. The gift was from fees they earned in that litigation and the law firm which made the gift is not currently involved in tobacco litigation.

Open Access This article is distributed under the terms of the Creative Commons Attribution Noncommercial License which permits any noncommercial use, distribution, and reproduction in any medium, provided the original author(s) and source are credited.

\section{References}

1. American Cancer Society (2009) Cancer facts and figures 2009. American Cancer Society, Atlanta, GA, p v

2. Thun MJ, Heath CW Jr (1997) Changes in mortality from smoking in two American Cancer Society prospective studies since 1959. Prev Med 26(4):422-426

3. Thun MJ, Henley SJ, Burns D, Jemal A, Shanks TG, Calle EE (2006) Lung cancer death rates in lifelong nonsmokers. J Natl Cancer Inst 98(10):691-699

4. Thun MJ, Hannan LM, Adams-Campbell LL, Boffetta P, Buring JE, Feskanich D et al (2008) Lung cancer occurrence in neversmokers: an analysis of 13 cohorts and 22 cancer registry studies. PLoS Med 5(9):e185

5. Doll R, Peto R, Wheatley K, Gray R, Sutherland I (1994) Mortality in relation to smoking: 40 years' observations on male British doctors. BMJ 309(6959):901-911

6. National Cancer Institute (U.S.) (2001) Risks associated with smoking cigarettes with low machine-measured yields of tar and nicotine. U.S. Dept. of Health and Human Services, Public Health 
Service, National Institutes of Health, National Cancer Institute, Bethesda, MD

7. Wynder EL, Muscat JE (1995) The changing epidemiology of smoking and lung cancer histology. Environ Health Perspect 103(Suppl 8):143-148

8. Hoffmann D, Hoffmann I (1997) The changing cigarette, 1950-1995. J Toxicol Environ Health 50(4):307-364

9. Knoke JD, Burns DM, Thun MJ (2008) The change in excess risk of lung cancer attributable to smoking following smoking cessation: an examination of different analytic approaches using CPS-I data. Cancer Causes Control 19(2):207-219

10. Knoke JD, Shanks TG, Vaughn JW, Thun MJ, Burns DM (2004) Lung cancer mortality is related to age in addition to duration and intensity of cigarette smoking: an analysis of CPS-I data. Cancer Epidemiol Biomark Prev 13(6):949-957

11. Burns DM, Lee LL, Gilpin B, Tolley HD, Vaughn J, Shanks T (1997) Cigarette smoking behavior in the United States. In: Burns DM, Garfinkel L, Samet JM (eds) Changes in cigarette related disease risks and Their Implications for Prevention and Control. National Cancer Institute, Bethesda, pp 13-112

12. Anderson CM, Burns DM, Dodd K, Feuer EJ (2010) Birth cohort specific estimates of smoking behaviors for the U.S. population risk analysis. Submitted

13. Mannino DM, Ford E, Giovino GA, Thun M (2001) Lung cancer mortality rates in birth cohorts in the United States from 1960 to 1994. Lung Cancer 31(2-3):91-99

14. Pinsky PF, Miller A, Kramer BS, Church T, Reding D, Prorok P et al (2007) Evidence of a healthy volunteer effect in the prostate, lung, colorectal, and ovarian cancer screening trial. Am J Epidemiol 165(8):874-881

15. Thun MJ, Lally CA, Flannery JT, Calle EE, Flanders WD, Heath CW Jr (1997) Cigarette smoking and changes in the histopathology of lung cancer. J Natl Cancer Inst 89(21):1580-1586

16. Zheng T, Holford TR, Boyle P, Chen Y, Ward BA, Flannery J et al (1994) Time trend and the age-period-cohort effect on the incidence of histologic types of lung cancer in Connecticut, 1960-1989. Cancer 74(5):1556-1567

17. Burns DM, Anderson CM, Gray N (2011) Do changes in cigarette design influence the rise in adenocarcinoma of the lung? Cancer Causes Control 22(1):13-22

18. Kenfield SA, Wei EK, Stampfer MJ, Rosner BA, Colditz GA (2008) Comparison of aspects of smoking among the four histological types of lung cancer. Tob Control 17(3):198-204
19. Bennett VA, Davies EA, Jack RH, Mak V, Moller H (2008) Histological subtype of lung cancer in relation to socio-economic deprivation in South East England. BMC Cancer 8:139

20. Swartz JB (1992) Use of a multistage model to predict time trends in smoking induced lung cancer. J Epidemiol Community Health 46(3):311-315

21. Whittemore AS (1988) Effect of cigarette smoking in epidemiological studies of lung cancer. Stat Med 7(1-2):223-238

22. Tolley HD, Crane L, Shipley N (1991) Smoking prevalence and lung cancer death rates. In: Shopland D, Burns DM, Samet JM, Gritz ER (eds) Strategies to Control Tobacco Use In the United States: A blueprint for public health action in the 1990s. National Cancer Institute, Bethesda, MD, pp 75-144

23. Peto R (1986) Influence of dose and duration of smoking on lung cancer rates. IARC Sci Publ 74:23-33

24. Wingo PA, Ries LA, Giovino GA, Miller DS, Rosenberg HM, Shopland DR et al (1999) Annual report to the nation on the status of cancer, 1973-1996, with a special section on lung cancer and tobacco smoking. J Natl Cancer Inst 91(8):675-690

25. Edwards BK, Ward E, Kohler BA, Eheman C, Zauber AG, Anderson RN et al (2009) Annual report to the nation on the status of cancer, 1975-2006, featuring colorectal cancer trends and impact of interventions (risk factors, screening, and treatment) to reduce future rates. Cancer 116(3):544-573

26. Flanders WD, Lally CA, Zhu BP, Henley SJ, Thun MJ (2003) Lung cancer mortality in relation to age, duration of smoking, and daily cigarette consumption: results from Cancer Prevention Study II. Cancer Res 63(19):6556-6562

27. Hoffmann D, Rivenson A, Hecht SS (1996) The biological significance of tobacco-specific N-nitrosamines: smoking and adenocarcinoma of the lung. Crit Rev Toxicol 26(2):199-211

28. Gower S, Hammond D (2007) CSP deposition to the alveolar region of the lung: implications of cigarette design. Risk Anal 27(6):1519-1533

29. Thun MJ, Burns DM (2001) Health impact of "reduced yield" cigarettes: a critical assessment of the epidemiological evidence. Tob Control 10(Suppl 1):i4-i11 